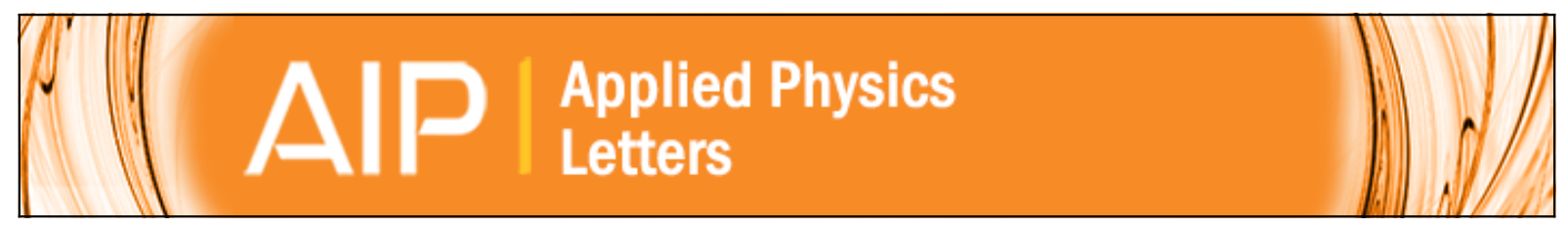

\title{
Exciton transitions in tetrapod-shaped CdTe nanocrystals investigated by photomodulated transmittance spectroscopy
}

Davide Tarì, Milena De Giorgi, Pier Paolo Pompa, Luigi Carbone, Liberato Manna, Stefan Kudera, and Roberto Cingolani

Citation: Applied Physics Letters 89, 094104 (2006); doi: 10.1063/1.2335801

View online: http://dx.doi.org/10.1063/1.2335801

View Table of Contents: http://scitation.aip.org/content/aip/journal/apl/89/9?ver=pdfcov

Published by the AIP Publishing

\section{Articles you may be interested in}

Improved photovoltaic performance of bilayer heterojunction photovoltaic cells by triplet materials and tetrapodshaped colloidal nanocrystals doping

Appl. Phys. Lett. 95, 043101 (2009); 10.1063/1.3186074

Modification of emission of CdTe nanocrystals by the local field of Langmuir-Blodgett colloidal photonic crystals J. Appl. Phys. 104, 103118 (2008); 10.1063/1.2981087

Radiative recombination dynamics in tetrapod-shaped CdTe nanocrystals: Evidence for a photoinduced screening of the internal electric field

Appl. Phys. Lett. 92, 191905 (2008); 10.1063/1.2924306

Size Dependent Photomodulated Transmission Spectroscopy of CdTe Tetrapodshaped Nanocrystals AIP Conf. Proc. 893, 1063 (2007); 10.1063/1.2730264

Optical properties of tetrapod-shaped CdTe nanocrystals

Appl. Phys. Lett. 87, 224101 (2005); 10.1063/1.2130727
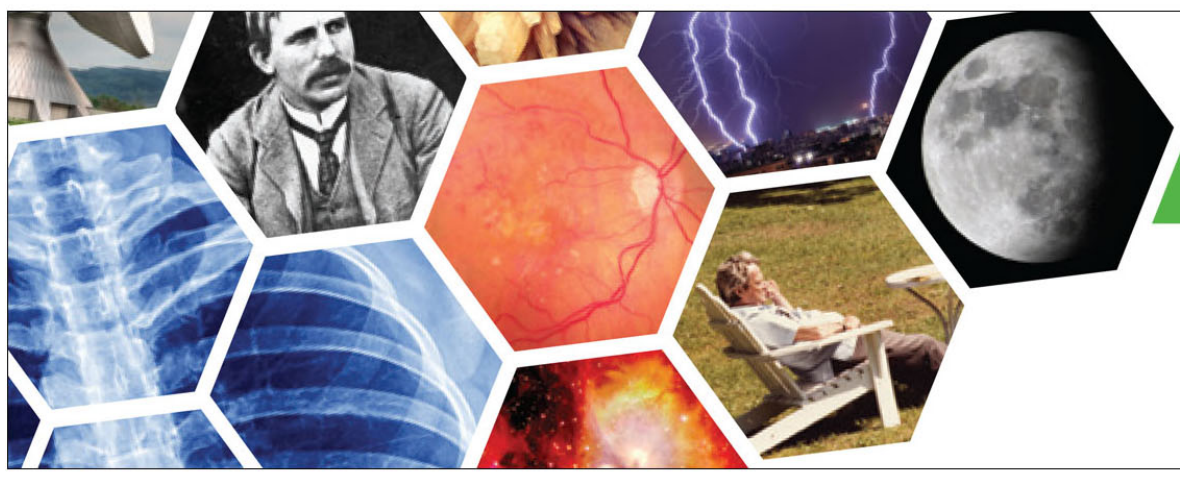

\section{SUBSCRIBE TO \\ physics today}




\title{
Exciton transitions in tetrapod-shaped CdTe nanocrystals investigated by photomodulated transmittance spectroscopy
}

\author{
Davide Tarì, a) Milena De Giorgi, Pier Paolo Pompa, Luigi Carbone, Liberato Manna, \\ Stefan Kudera, and Roberto Cingolani \\ National Nanotechnology Laboratory, INFM-CNR, clo DistrettoTecnologico ISUFI, Università di Lecce, Via \\ per Arnesano, 73100 Lecce, Italy
}

(Received 1 March 2006; accepted 20 June 2006; published online 30 August 2006)

\begin{abstract}
The excitonic nature of the optical transitions in tetrapod-shaped colloidal CdTe nanocrystals is assessed by means of photomodulated transmittance spectroscopy. The line-shape analysis of the photomodulation transmittance spectra indicates the photoinduced Stark effect as the dominant modulation mechanism, and the presence of excitonic transitions even at room temperature, with an exciton binding energy of about $25 \mathrm{meV}$, larger than the bulk value. (C) 2006 American Institute of Physics. [DOI: 10.1063/1.2335801]
\end{abstract}

Colloidal nanocrystals are promising materials in different fields of nanoscience and nanotechnology. Spherical ${ }^{1}$ and rod-shaped ${ }^{2}$ nanocrystals of different sizes, for instance, have been proposed for applications in biological tagging ${ }^{3}$ as additives in plastic solar cells ${ }^{4,5}$ and optoelectronic devices. ${ }^{6}$ More advanced applications can be envisaged if we consider nanocrystals with more complex morphology, such as tetrapods. ${ }^{7}$ A tetrapod-shaped nanocrystal is formed by four arms that branch out at tetrahedral angles from a central region. Due to their shape, tetrapods are appealing for optoelectronic devices, where charge migration phenomena represent a critical parameter. ${ }^{5,8}$ Cui et al., for instance, have employed a single electron transistor approach, in which three arms of a CdTe tetrapod-shaped nanocrystal are contacted with metal electrodes to understand how charge carriers move through branching points. ${ }^{9}$ Although the carrier dynamics in these branched structures is of great interest, a few experimental and theoretical works have exhaustively investigated the complicated band structure of a tetrapod. ${ }^{10}$ Some of us studied in a previous work ${ }^{11}$ the carrier confinement in CdTe tetrapod by means of absorption and photoluminescenc measurements as a function of the tetrapod size. The observed optical transitions were assigned to tetrapod excited states by a theoretical calculation based on the envelopefunction approximation.

In this letter, we assess the excitonic nature of the electronic transitions in CdTe tetrapod-shaped nanocrystals by photomodulated transmittance measurements (PMT) performed at various temperatures, evidencing the excitonic resonances even at room temperature and elucidating the origin of the modulation mechanism. The photomodulated transmittance ${ }^{12}$ is a nondestructive technique in which a contactless electric field modulation is produced by a laser beam. Due to the suppression of substrate and continuum state contributions, the PMT shows an intrinsic enhanced sensitivity compared to linear absorption measurements. In the past, several groups have applied modulation spectroscopy to investigate the origin of the built-in dipole moment in CdSe spherical and rod-shaped nanocrystals. ${ }^{13}$ Only recently, photomodulated transmittance spectroscopy has been

a) Author to whom correspondence should be addressed; electronic mail: davide.tari@unile.it used to characterize the $E_{0}$ critical-point structure in $\mathrm{ZnO}$ nanowires. ${ }^{14}$

In this work, photomodulation transmittance was carried out on two classes of tetrapod samples characterized by different sizes and aspect ratios. The synthesis of CdTe tetrapods was performed according to the protocol described by Carbone et al. ${ }^{15}$ The size of the samples was assessed by transmission electron microscopy (TEM) analysis [Figs. 1(a) and 1(b)], with accuracy better than 5\%. The TEM images show a good homogeneity of the two samples and a rather narrow distribution of arm diameters $(d)$ and lengths $(l)$, whose average values were found to be $d=7.2 \mathrm{~nm}$ and $l$ $=50 \mathrm{~nm}$ for sample SAM 1 and $d=5.7 \mathrm{~nm}$ and $l=30 \mathrm{~nm}$ for SAM2.

For the temperature-dependent PMT measurements the tetrapod samples were dispersed in a polystyrene film and the film was attached on a sapphire plate onto the cold finger of a cryostat. A $200 \mathrm{~W}$ halogen tungsten lamp was dispersed by a $0.5 \mathrm{~m}$ monochromator, producing the probe beam, whereas a $5 \mathrm{~mW}$ blue line of an $\mathrm{Ar}^{+}$laser (458 nm), chopped at a frequency of $115 \mathrm{~Hz}$, was used as the modulator beam. Both the ac and dc parts of the transmitted light, proportional to the transmittance changes $\Delta T$ and to the transmittance $T$,
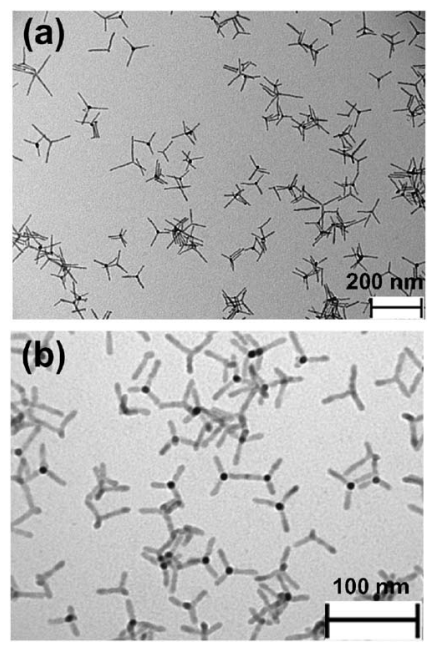

FIG. 1. Transmission electron micrographs (TEM) of the two CdTe tetrapod samples: SAM1 (a) and SAM2 (b). In each sample the central dark spot is due to the tetrapod arm towards the incoming electron beam. 


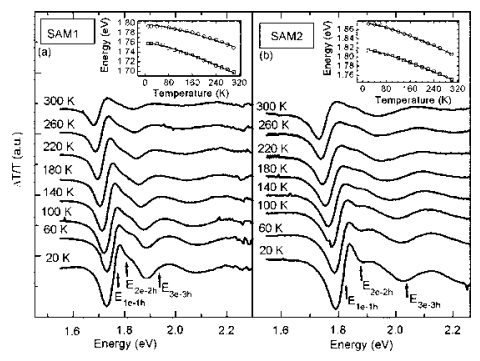

FIG. 2. Temperature-dependent photomodulation transmission spectra of SAM1 (a) and SAM2 (b). The different curves are shifted for clarity. The vertical arrows at low temperature indicate the position of the three transitions observed. The inset shows the energy peak fitted by the Varshni function for $E_{1 e-1 h}$ and $E_{2 e-2 h}$ transitions.

respectively, were detected by a $\mathrm{Si}$ photodiode using a lock-in amplifier.

Figures 2(a) and 2(b) show the photomodulation transmission spectra of the two samples recorded in the range of 20-300 K. The PMT spectra exhibit several resonances corresponding to different electron-hole transitions. The spectral line shape was accurately calculated (see below) to determine the transition energies marked by the vertical arrows at low temperature in Figs. 2(a) and 2(b). In both samples we could identify three main optical transitions, labeled $E_{1 e-1 h}$, $E_{2 e-2 h}$, and $E_{3 e-3 h}$, whose spectral features were size and temperature dependent. In the smaller tetrapod sample, the whole spectrum is blueshifted with respect to the larger tetrapod sample, as expected from the stronger quantum size effect. In fact, the energy of the ground level transition increases from $1.758 \mathrm{eV}$ in SAM1 to $1.814 \mathrm{eV}$ in SAM2. In addition, the energy spacing between the two lower energy features is remarkably larger in the smaller sample (about $60 \mathrm{meV}$ ) as compared to the larger sample (about $40 \mathrm{meV}$ ).

At higher temperatures, the overall signal intensity smears out, with a large thermal broadening as usually observed in the absorption spectra. In addition the spectral features redshift with increasing temperature [see inset of Figs. 2(a) and 2(b)] following Varshni's equation: ${ }^{16}$

$$
E_{g}(T)=E_{g}(0)-\frac{a T^{2}}{b+T},
$$

where $E_{g}$ is the energy gap of the material, $T$ is the temperature, and $a$ and $b$ are Varshni's coefficients. The best fit for both samples is found for values of $a$ and $b$ which are in good agreement with the typical values reported for CdTe bulk. $^{17}$

In order to understand the nature of the energy states as revealed from the PMT spectra, we need to analyze the single transition behavior induced by an external perturbation field, as a function of the parameters that govern the modulation mechanisms. The normalized transmittance change $(\Delta T / T)$ can be expressed by the following formula: ${ }^{18}$

$$
\Delta T / T=\alpha \Delta \varepsilon_{1}+\beta \Delta \varepsilon_{2},
$$

where $\alpha\left(\varepsilon_{1}, \varepsilon_{2}\right)$ and $\beta\left(\varepsilon_{1}, \varepsilon_{2}\right)$ are the Seraphin coefficients, and $\Delta \varepsilon_{1}$ (related to the reflectance variations) and $\Delta \varepsilon_{2}$ (related to the absorption variations) are the changes in the real and the imaginary parts of the dielectric function generated by the modulation beam, respectively. Since in the PMT the perturbation of the real part of the dielectric function is neglected, ${ }^{19}$ only $\Delta \varepsilon_{2}$ contributes to the PM'T signal. $\Delta \varepsilon_{2}$ can

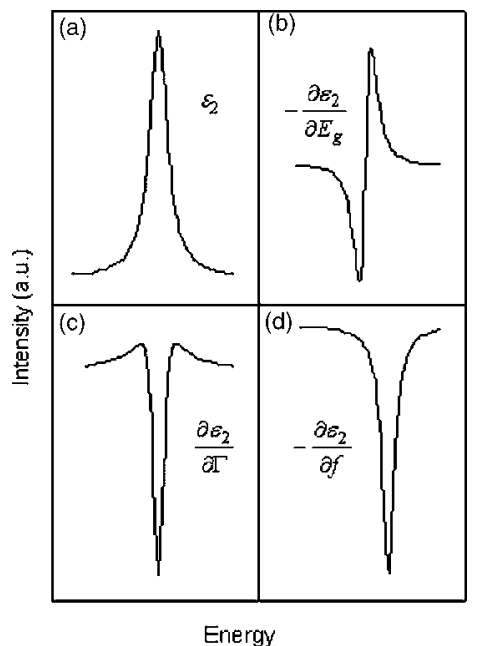

FIG. 3. (a) Illustration of the imaginary part of the dielectric function. [(b)(d)] Single contributions of the PMT spectra line shape due to transition energy modulation (b), broadening modulation (c), and oscillator strength modulation (d). The (b) and (d) functions have been multiplied by -1 to compare with the line shape of the expected curves.

be written as a function of the broadening $(\Gamma)$, energy gap $\left(E_{g}\right)$, and oscillator strength $(f):^{20}$

$$
\Delta \varepsilon_{2}=\frac{\partial \varepsilon_{2}}{\partial E_{g}}\left(\frac{\partial E_{g}}{\partial P}\right)+\frac{\partial \varepsilon_{2}}{\partial \Gamma}\left(\frac{\partial \Gamma}{\partial P}\right)+\frac{\partial \varepsilon_{2}}{\partial f}\left(\frac{\partial f}{\partial P}\right) .
$$

The bracketed coefficients, which depend on the modulation intensity (indicated with $P$ ), give the weight of the different modulation mechanisms for each transition. Usually, for temperatures below $150 \mathrm{~K}$ the PMT spectrum is described by a Lorentzian line shape, whereas at higher temperature a Gaussian absorption profile is more suitable. In our experiments, instead, we found that a Lorentzian profile, ${ }^{21}$

$$
\frac{\Delta T}{T}(E)=\operatorname{Im}\left[\frac{A e^{i \Theta}}{\left(E-E_{g}+i \Gamma\right)^{n}}\right],
$$

gives the best line-shape fitting even at room temperature. In Eq. (4) the amplitude $A$ (which is oscillator strength dependent), the broadening $\Gamma$, the transition energy $E_{g}$, and the phase angle $\Theta$ have been employed as free parameters in the fit. In Eq. (4) the exponential coefficient $n$ can take three discrete values, depending on the type of optical transition. These are $n=2.5$ and $n=3$ for a three-dimensional electron transition and two-dimensional electron transition, respectively, while $n=2$ is required to fit an excitonic transition in a highly confined systems. The best fit of our PMT spectra is obtained by using a coefficient $n=2$ in the whole temperature range of interest $(20-300 \mathrm{~K})$, indicating the occurrence of excitonic resonances up to room temperature. It is worth noting that the obtained values of $\Gamma$ and $E_{g}$ are in good agreement with those estimated by the fit of the absorption spectra. To support our conclusion we fitted PMT spectra when a coefficient $n$ equal to 2.5 or 3 is assumed. A fit error analysis has shown that these values for the coefficient $n$ are inadequate in our case. ${ }^{22}$

Figures 3(b)-3(d) display the expected PMT line shapes for the three different modulation mechanisms in Eq. (3), assuming the Lorentzian profile of $\varepsilon_{2}$.

Figures 4(a) and 4(b) display some representative fitting curves for two samples, at low temperature, using Eqs. (2)-(4). The excellent agreement between the best fit curves 


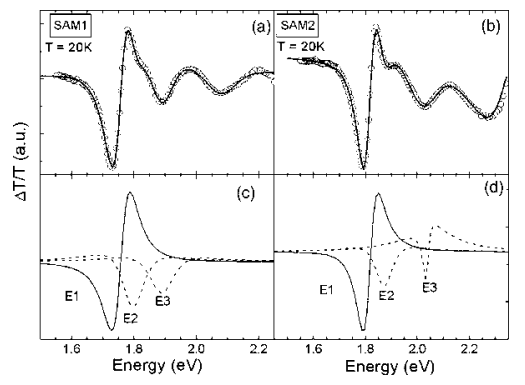

FIG. 4. [(a) and (b)] Example of the fit procedure carried out on the PMT spectra at low temperature for both samples; [(c) and (d)] contributions of single transitions derived from the total fitted PMT spectrum at low temperature for both samples.

and the measured line shape indicates that the PMT spectra originate primarily from the electric field modulation of the excitonic transitions rather than from band-to-band transitions, as normally observed in bulk materials. In spite of the relatively low exciton binding energy in $\mathrm{CdTe},{ }^{23}$ in the tetrapod structure we are able to observe exciton transitions even at high temperatures. This is attributed to the strong quantum confinement of the carriers that prevent a complete ionization of the exciton, suggesting an exciton binding energy value higher than the thermal energy at room temperature (about $25 \mathrm{meV}$ ).

In SAM1 the dispersive antisymmetric line shape of the $E_{1 e-1 h}$ transition suggests that the first term in Eq. (3) prevails over the other two. We assume that the main effect of the built-in field variation is the redshift of the energy of the confined state by photoinduced electric field through a photoinduced Stark effect. ${ }^{24}$ In several previous works different effects have been proposed to explain the modulation of the dielectric function. ${ }^{25,26}$ However, in our study the experimental evidence and the peculiar structure of the samples suggest a different origin of the modulation mechanism, i.e., a photoinduced Stark effect. We believe that in laser off conditions a built-in field originates from the selective coordination of the surfactants, which completely passivates the $\mathrm{Cd}$ atoms and only partly the Te dangling bonds, which therefore act as hole traps. ${ }^{27}$ When the modulation beam is on, the photogenerated carriers are trapped on the surface sites, resulting in a screening of the built-in field. For the $E_{2 e-2 h}$ and the $E_{3 e-3 h}$ transitions of SAM1, we can assume that both the oscillator strength and the broadening are responsible for the modulation, as they exhibit symmetric spectral forms which are in qualitative agreement with theoretical predictions. Similar conclusions can be drawn for SAM2, but for the $E_{3 e-3 h}$ transition, for which a considerable contribution of the energetic term is needed to describe the slight asymmetry with respect to the base line.

In conclusion, we have explained the excitonic nature of the optical transitions in CdTe tetrapod-shaped nanocrystals by performing a photomodulation transmittance study at various temperatures. The measured spectra show the expected trends: (i) the broadening and the redshift of the curves when the temperature increases and (ii) the blueshift of the features and the enhancement of the energy spacing between optical transition when the size of sample decreases. By an accurate line-shape analysis, we have been able to demonstrate the excitonic nature of the PMT resonances even at room temperature and to identify the photoinduced Stark effect as the dominant modulation mechanism.
The authors want to thank G. Rainò for useful discussion. They also thank the expert technical help of P. Cazzato. This work has been partially supported by the SA-NANO European project Contract No. STRP013698, and by the MIUR-FIRB project.

${ }^{1}$ A. L. Efros, M. Rosen, M. Kuno, M. Nirmal, D. J. Norris, and M. Bawendi, Phys. Rev. B 54, 4843 (1996); D. J. Norris, A. L. Efros, M. Rosen, and M. G. Bawendi, ibid. 53, 16347 (1996).

${ }^{2}$ J. T. Hu, L. W. Wang, L. S. Li, W. D. Yang, and A. P. Alivisatos, J. Phys. Chem. B 106, 2447 (2002); J. T. Hu, L. S. Li, W. D. Yang, L. Manna, L. W. Wang, and A. P. Alivisatos, Science 292, 2060 (2001).

${ }^{3}$ M. Bruchez, M. Moronne, P. Gin, S. Weiss, and A. P. Alivisatos, Science 281, 2013 (1998). W. J. Parak, D. Gerion, T. Pellegrino, D. Zanchet, C. Micheel, S. C. Williams, R. Boudreau, M. A. Le Gros, C. A. Larabell, and A. P. Alivisatos, Nanotechnology 14, R15 (2003).

${ }^{4}$ W. U. Huynh, J. J. Dittmer, and A. P. Alivisatos, Science 295, 2425 (2002); D. J. Milliron, I. Gur, and A. P. Alivisatos, MRS Bull. 30, 41 (2005).

${ }^{5}$ B. Q. Sun, E. Marx, and N. C. Greenham, Nano Lett. 3, 961 (2003).

${ }^{6}$ D. L. Klein, R. Roth, A. K. L. Lim, A. P. Alivisatos, and P. L. McEuen, Nature (London) 389, 699 (1997); N. Tessler, V. Medvedev, M. Kazes, S. H. Kan, and U. Banin, Science 295, 1506 (2002); H. Htoon, J. A. Hollingworth, A. V. Malko, R. Dickerson, and V. I. Klimov, Appl. Phys. Lett. 82, 4776 (2003).

${ }^{7}$ L. Manna, D. J. Milliron, A. Meisel, E. C. Scher, and A. P. Alivisatos, Nat. Mater. 2, 382 (2003).

${ }^{8}$ P. Peng, D. J. Milliron, S. M. Hughes, J. C. Johnson, A. P. Alivisatos, and R. J. Saykally, Nano Lett. 5, 1809 (2005).

${ }^{9}$ Y. Cui, U. Banin, M. T. Bjork, and A. P. Alivisatos, Nano Lett. 5, 1519 (2005).

${ }^{10}$ J. B. Li and L. W. Wang, Nano Lett. 3, 1357 (2003).

${ }^{11}$ D. Tari, M. De Giorgi, F. Della Sala, L. Carbone, R. Krahne, L. Manna, R. Cingolani, S. Kudera, and W. J. Parak, Appl. Phys. Lett. 87, 224101 (2005).

${ }^{12}$ P. Handler, A. Frova, F. A. Germano, and D. E. Aspnes, Phys. Rev. 145, 575 (1966).

${ }^{13}$ A. Sacra, D. J. Norris, C. B. Murray, and M. G. Bawendi, J. Chem. Phys. 103, 5236 (1995); V. L. Colvin, K. L. Cunningham, and A. P. Alivisatos, ibid. 101, 7122 (1994).

${ }^{14}$ S. Ozaki, T. Tsuchiya, Y. Inokuchi, and S. Adachi, Phys. Status Solidi A 202, 1325 (2005).

${ }^{15}$ L. Carbone, S. Kudera, E. Carlino, W. J. Parak, C. Giannini, R. Cingolani, and L. Manna, J. Am. Chem. Soc. 128, 748 (2006).

${ }^{16}$ V. P. Varshni, Physica (Amsterdam) 34, 149 (1967); J. Perez-Conde, A. K. Bhattacharjee, M. Chamarro, P. Lavallard, V. D. Petrikov, and A. A. Lipovskii, Phys. Rev. B 64, 113303 (2001).

${ }^{17}$ U. Pal, J. L. H. Perez, J. Piqueras, and E. Dieguez, Mater. Sci. Eng., B 42, 297 (1996).

${ }^{18}$ D. E. Aspnes, Optical Properties of Solids, edited by M. Balkanski (North-Holland, Amsterdam, 1980), Vol. 2, Chap. 4A, p. 109.

${ }^{19}$ K. L. Stokes and P. D. Persans, Phys. Rev. B 54, 1892 (1996).

${ }^{20}$ O. J. Glembocki, W. T. Beard, and B. V. Shanabrook, Phys. Rev. B 35, 2540 (1987); U. Woggon, S. V. Bogdanov, O. Wind, K. H. Schlaad, H. Pier, C. Klingshirn, P. Chatziagorastou, and H. P. Fritz, ibid. 48, 11979 (1993).

${ }^{21}$ J. Misiewicz, P. Sitarek, and G. Sek, Opto-Electron. Rev. 8, 1 (2000); D. E. Aspnes, Surf. Sci. 37, 418 (1973).

${ }^{22} \mathrm{We}$ have performed an error fit analysis by using three different values of the $n$ coefficient. For example, we report the fit standard error [root mean square error (RMSE)] for the fit curves of the PMT spectrum of SAM2 at $20 \mathrm{~K}$. For the coefficient $n$ equal to 2, 2.5, and 3, we obtained a RMSE of $0.00133,0.00412$, and 0.1041 , respectively, confirming the best fit conditions for coefficient value $n=2$.

${ }^{23}$ Z. H. Yu, S. G. Hofer, N. C. Giles, T. H. Myers, and C. J. Summers, Phys. Rev. B 51, 13789 (1995).

${ }^{24}$ D. J. Norris, A. Sacra, C. B. Murray, and M. G. Bawendi, Phys. Rev. Lett. 72, 2612 (1994).

${ }^{25}$ D. S. Ginger, A. S. Dhoot, C. E. Finlayson, and N. C. Greenham, Appl. Phys. Lett. 77, 2816 (2000).

${ }^{26}$ L. T. Klauder and J. G. Gay, Phys. Rev. 172, 811 (1968).

${ }^{27}$ S. Pokrant and K. B. Whaley, Eur. Phys. J. D 6, 255 (1999); M. Shim and P. Guyot-Sionnest, I Chero, Phys. M11, 6955 (1999). ditions. Downloaded to IP: 